\title{
Stepping up: the just released new impact factor 2015
}

\author{
Daniela Reich-Erkelenz ${ }^{1} \cdot$ Andrea Schmitt $^{2} \cdot$ Peter Falkai $^{2}$
}

Published online: 18 July 2016

(C) Springer-Verlag Berlin Heidelberg 2016

We are proud of opening this issue with awesome news: ISI has just released the new impact factor list for 2015, according to which European Archives of Psychiatry and Clinical Neuroscience (EAPCN) for the fourth time in a row has risen its impact factor and now has achieved a rank of 4.113, thus for the first time negotiating the hurdle of 4. EAPCN has scaled a rating notably above the average impact factor of both areas Clinical Neurology (Ø 2.920) and Psychiatry ( $\varnothing$ 2.987). At the same time, our journal improved its position to 36 in Clinical Neurology (out of 192) and 30 in the psychiatric area (out of 140), thus accomplishing the top ranking quarter of all journals in both fields.
Andrea Schmitt

Andrea.Schmitt@med.uni-muenchen.de

1 Institute of Psychiatric Phenomics and Genomics, Ludwig-Maximilians-University Munich, Nußbaumstr. 7, 80336 Munich, Germany

2 Department of Psychiatry and Psychotherapy, LudwigMaximilians-University Munich, Nußbaumstr. 7, 80336 Munich, Germany

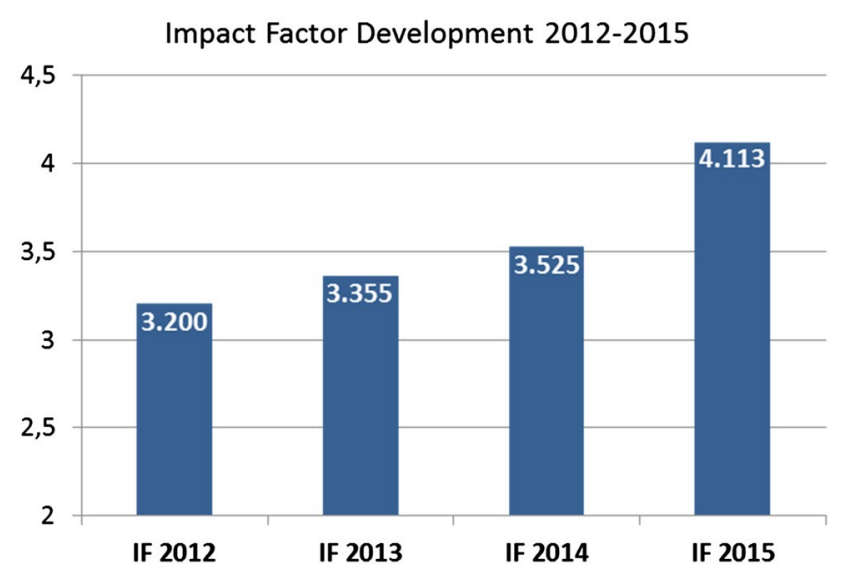

Besides, EAPCN has taken into account author's interests by markedly shortening the days until first decision by nearly one-third - the original duration of about 50 days now averages 1 month. The same applies to the general review process in its entirety by shortening the former duration of nearly half a year to $<2$ months for all submissions, the review process until acceptance now averages around 4 months. This development is mirrored by an increasing number of authors interested in placing their manuscripts in this journal: In 2015, we received around $20 \%$ more submissions than in 2014, which might afford slightly raising the comparably moderate rejection rate in the future.

As to the previously determined interest in clinical studies, EAPCN has strengthened its respective focus, namely studies preferably entailing prospective data, large sample sizes, and effects of modern therapeutic strategies. An additional emphasis has been set on neurobiological insights into psychiatric diseases which contribute to unravelling pathophysiological aspects. A look on the best cited papers in 2013 and 2014 affirms topics on innovative therapeutic strategies to meet readers' preferences: Most citations 
reached an article on glutamate modulators as potential therapeutic drugs in schizophrenia and affective disorders. Further examples of well-cited papers show attentiveness to topics dealing with comorbidities, be it a long-term study on the relevance of physical comorbidity in schizophrenia or a systematic review and meta-analysis of anxiety and depression comorbidities in irritable bowel syndrome. As to neurobiological questions for example, the thalamocortical connectivity during resting state in schizophrenia was of interest.
This success could only be attained by the joint effort of our reviewers and editors who with formidable input contributed to this achievement and whom we want to express our sincere gratitude. We also, of course, want to acknowledge all authors, who honored us by sending their manuscripts and added important knowledge to our field of expertise. 